\title{
Mechanistic study of aerosol dry deposition on vegetated canopies
}

\author{
A. Petroff \\ I.R.S.N., C.E. Cadarache, Bât. 159, 13108 Saint Paul-lez-Durance, France \\ e-mail: alexandre.petroff@irsn.fr
}

\begin{abstract}
Aerosol dry deposition onto agricultural and forest areas is investigated. Its estimation is still uncertain, particularly for rough surfaces and sub-micronic aerosols. In fact models used in radiological risk or air quality assessment studies are highly empirical and fail to reproduce the results of the most recent measurement campaigns. Therefore a theoretical framework, based on a mechanistic description, has been developed. The proposed approach consists in two steps. First, interaction between aerosols and foliar surface is formulated by using a set of parameters, which are defined on the local scale of one foliar element. In the second step, the collective effect of the foliage is taken into account through statistical distribution of these parameters. The model integrates the three main factors governing aerosol dry deposition. These are local aerodynamic characteristics of the flow within the canopy, aerosol mechanisms governing the deposition, and canopy structural and morphological properties. Physical processes considered in the study are inertial impaction, gravitational settling and Brownian diffusion. The canopy characteristics considered are spatial distribution, orientation and micro-structure of foliar surfaces. The applicability of the model is demonstrated on an existing measurement campaign.
\end{abstract}

\section{INTRODUCTION}

The context of this study is the radiological risk assessment in terrestrial environment exposed to an airborne particulate contamination. Risk assessment quality depends directly on quality of dry deposition forecast. The aim of this study is a better estimate of aerosol dry deposition, particularly for sub-micronic aerosols, since it has been proved that they were responsible for radio-nuclide long range transport after Chernobyl accident [1].

Transport models currently used in risk assesment (or in air quality prediction) estimate deposition flux on a vegetated surface through a bulk parameter called deposition velocity. Theoretically it depends on aerosol size distribution, flow dynamics and canopy characteristics. The existing models, i.e. [2], highly empirical, fail to reflect recent measurements made in forested canopies [3-5].

In order to improve dry deposition forecast, the transport and the deposition are considered together within the canopy. The developed method is presented, then its results are compared with experimental data.

\section{THEORETICAL CONSIDERATIONS}

In a first step, the aerosol transport and deposition equation is derived following the recent averaging method used in canopy turbulence studies, i.e. [6]. In a second step, deposition terms are modelled following a new approach. In this approach, each vegetated obstacle, mainly leaves, is considered as 
separated from the rest of the canopy. Each aerosol deposition mechanism is evaluated with local parameters. Deposition occurring in a representative volume, containing a large number of leaves, is then deduced using a statistical treatment based on those parameters.

\subsection{Aerosol transport in canopy}

The main model hypotheses are as follows. Concerning the canopy, the only considered organs are leaves, because other organs surface is negligible. Concerning aerosols, convection, sedimentation and diffusion processes are taken into account in the transport equation. Aerosol concentration is named $\chi$. Inside the canopy, the flow is turbulent.

The method used to derive the transport equation is the same as in turbulent studies [6]. The canopy is viewed as a weakly porous medium. An Eulerian approach is followed. A vegetation representative volume, $V$, is defined for spatial averaging. Two averaging operations, according to time and space, are introduced. For all $\phi$ (scalar or vector), these are:

$$
\bar{\phi}(t)=\frac{1}{T} \int_{t-T / 2}^{t+T / 2} \phi(\tau) d \tau \quad \text { and } \quad\langle\phi(\vec{x})\rangle=\frac{1}{V} \iiint_{\vec{M} \in V} \phi(\vec{x}+\vec{M}) d^{3} \omega
$$

where $T$ is the sampling period. The aerosol equation is deduced from averaging operations to:

$$
\frac{\partial\langle\bar{\chi}\rangle}{\partial t}+\vec{\nabla} \cdot\left[\langle\overline{\vec{u}}\rangle\langle\bar{\chi}\rangle-W_{S}\langle\bar{\chi}\rangle \overrightarrow{e z}+\left\langle\overline{\chi^{\prime} \vec{u}^{\prime}}\right\rangle+\left\langle\bar{\chi}^{\prime \prime} \overrightarrow{\vec{u}}^{\prime \prime}\right\rangle\right]=d D
$$

where $\vec{u}$ is the instantaneous flow velocity, $W_{S}$ is the aerosol settling velocity, $\overline{\chi^{\prime}} \overrightarrow{u^{\prime}}$ is the aerosol turbulent flux, which will be modelled with a first order closure model. The last term on the left-hand side is a flux generated by spatial fluctuations. It will be neglected compared with the turbulent flux, as in aerodynamic studies [7]. On the right-hand side lies the overall deposition density, occurring in the representative volume. It is written as a sum of all deposition mechanism contribution:

$$
d D=\sum_{\alpha=1}^{m} d D^{\alpha}
$$

where $m$ is the number of mechanisms and $d D^{\alpha}$ the deposition flux density associated with the $\alpha^{\text {th }}$ mechanism. These terms need to be modelled. The method used will now be exposed.

\subsection{Deposition model}

We propose [8] original approach to model the deposition in a representative volume. It consists in two steps. First, single leaf deposition is estimated in a deterministic way. It means that it is expressed through local parameters like foliar area, surface orientation or local flow velocity. Second, this deposition is integrated over entire foliage contained in the representative volume. For that purpose, a leaf statistical distribution is built on these local parameters.

The main hypotheses are as follows. The surface is a perfect sink for particles. No bounce-off or blow-off is allowed after deposition. Each leaf can be considered as isolated from the rest of the foliage. No aerodynamic interaction between leaves is taken into account. The leaves number in the representative volume is supposed to be large enough to define the required distribution functions. 
Deposition occurring on a single leaf (total area $s$ ) is expressed through a volume averaged concentration and an effective deposition velocity, $v^{\alpha}$, which is mechanism specific:

$$
d^{\alpha}=-v^{\alpha} s\langle\bar{\chi}\rangle
$$

The $q$ physical parameters affecting this deposition are called

$$
\boldsymbol{\psi}^{\alpha}=\left\{\psi_{1}^{\alpha}, \ldots, \psi_{q}^{\alpha}\right\} \quad \text { with } \quad d \boldsymbol{\psi}^{\alpha}=\prod_{j=1}^{q} d \psi_{j}^{\alpha}
$$

and the domain of variations of these parameters is called $D\left(\psi^{\alpha}\right)$. Leaf statistics based on these parameters are built, with $f^{\alpha}\left(\boldsymbol{\psi}^{\alpha}\right)$ the leaf number distribution function. The foliage area density, $a$, is defined as $a=C^{N} \bar{s}$ where $C^{N}$ is the leaf number concentration and $\bar{s}$ the averaged foliar area. The volume averaged deposition associated with the $\alpha^{\text {th }}$ mechanism is then expressed thanks to the foliar statistics:

$$
d D^{\alpha}=C^{N} \int_{D\left(\psi^{\alpha}\right)} d^{\alpha} f^{\alpha} d \boldsymbol{\psi}^{\alpha}=-a V^{\alpha}\langle\bar{\chi}\rangle
$$

Where $V^{\alpha}$ is the macroscopic deposition velocity which takes into account parameters variability in the representative volume. It is related to the local deposition velocity through the following relation:

$$
V^{\alpha}=\int_{D\left(\psi^{\alpha}\right)} \stackrel{S}{=} v^{\alpha} f^{\alpha} d \boldsymbol{\psi}^{\alpha}
$$

The transport/deposition equation has been derived within the canopy. An original deposition model, based on leaf statistics, has been presented. In order to improve this model, it will be applied to an existing experiments campaign.

\section{APPLICATION}

We propose to test our model on a grass canopy configuration. The existing experiments used [9] have been carried out in a wind tunnel with both artificial and natural grasses. The artificial canopy is made of sticky vertical and rigid strips (height $h_{c}=7.5 \mathrm{~cm}$ and averaged width $\bar{L}=0.5 \mathrm{~cm}$ ). The strips orientation distribution is uniform. Natural grass (Lolium multiflorium) is grown to height $h_{c}=6 \mathrm{~cm}$ (averaged width $\bar{L}=0.3 \mathrm{~cm}$ ).

Main part of experiments is made with Lycopodium spores tagged with radioactive markers (Stokes diameter $d_{p}=23.6 \mu \mathrm{m}$ and density $\rho_{p}=1.15 \mathrm{~g} . \mathrm{cm}^{-3}$ ). Other aerosols have also been employed ( $d_{p}=19,5,1$ and $\sim 0.08 \mu \mathrm{m}$ with the same density $1.05 \mathrm{~g} . \mathrm{cm}^{-3}$ ).

We will briefly present the simplified transport/deposition model, before exposing comparison with experimental results.

\subsection{Transport model}

It is assumed that the flow regime is quasi stationary over experiments duration and that longitudinal gradients are negligible against vertical gradients. Using such circumstances, equation (2) can be simplified and we obtain: 


$$
\begin{aligned}
& z_{R} \geq z>h_{c} \quad \frac{d}{d z}\left[D_{T} \frac{d \chi}{d z}\right]+W_{S} \frac{d \chi}{d z}=0 \\
& z \leq h_{c} \quad \frac{d}{d z}\left[D_{T} \frac{d \chi}{d z}\right]+W_{S} \frac{d \chi}{d z}-\left(a \sum_{\alpha} V_{\alpha}\right) \chi=0
\end{aligned}
$$

Where $h_{c}$ is a canopy height, $z_{R}$ is the reference height (usually taken as $2 h_{c}$ ) and $D_{T}$ the turbulent diffusivity of aerosol particles. Averaging symbols are omitted here for simplicity. It is assumed to be equal to the flow turbulent viscosity. Its expressions are:

$$
\begin{aligned}
& z_{R} \geq z>h_{c} \quad D_{T}(z)=\kappa u^{*}(z-d) \\
& z \leq h_{c} \quad D_{T}(z)=\kappa u^{*}\left(h_{c}-d\right) e^{-\sigma\left(1-\frac{z}{h_{c}}\right)}
\end{aligned}
$$

where $u^{*}$ is the friction velocity, $\kappa$ the von Kärman constant $(\sim 0.41), d$ the canopy displacement height, $\sigma$ is a constant usually taken between 2 and 3 for moderately dense canopies [10]. We choose $\sigma=3$. The underlying hypothesis of this canopy formulation is that the inertial layer bottom corresponds to the canopy top.

Equation (7) is implemented and solved numerically. The soil deposition flux is assumed to be driven by sedimentation. With this boundary condition, concentration and flux profiles within and above the canopy are obtained. If $F$ is the total deposition flux (over vegetation and soil), the canopy deposition velocity $V_{d}$ is defined by the relation:

$$
V_{d}=-\frac{F}{\chi\left(z_{R}\right)}
$$

\subsection{Deposition model}

For this application the only considered deposition mechanisms will be inertial impaction, sedimentation and Brownian diffusion. For each of them, the modelling approach described above is applied and leads to practical formulations of the macroscopic deposition velocity. The grass leaves are considered as plane surfaces.

\subsubsection{Inertial impaction}

The single leaf deposition velocity, $v^{I M}$, is linked to the horizontal averaged velocity $U$ and leaf orientation by the impaction efficiency $E^{I M}$ :

$$
v^{I M}=\frac{1}{2} E^{I M} U \sin \theta \cos \phi \quad \text { with } \quad E^{I M}=\left(\frac{\mathrm{St}}{\beta+\mathrm{St}}\right)^{2}
$$

where $\theta$ and $\phi$ are leaf inclination and azimuth angles, the $\beta$ constant is taken as 0.3 and St is the Stokes number, defined by $\mathrm{St}=\rho_{p} U d_{p}^{2} / 18 \mu_{a} L$ where $\mu_{a}$ is dynamic viscosity and $L$ leaf width. We suppose that leaf width, inclination and azimuth are the only random parameters. They are supposed to be independent. Width statistical distribution is chosen as uniform. Macroscopic deposition velocity is deduced from Eq. (6): 


$$
V^{I M}=\frac{3}{8} K_{\theta 1} K_{\phi} U \frac{\overline{\mathrm{St}}^{2}}{\beta^{2}}\left[2 \frac{\overline{\mathrm{St}}+\beta}{\overline{\mathrm{St}}+2 \beta}-\frac{\overline{\mathrm{St}}}{\beta} \ln \left(1+2 \frac{\beta}{\overline{\mathrm{St}}}\right)\right]
$$

where $\overline{\mathrm{St}}$ is the Stokes number built on the averaged leaf width, $K_{\theta 1}$ et $K_{\phi}$ two constants linked to the angle distribution by $K_{\theta 1}=\overline{\sin \theta}$ and $K_{\phi}=\overline{\cos \phi}$. For both canopies, the azimuth distribution is though to be uniform so $K_{\phi}=2 / \pi$. Artificial grass is vertical, so $K_{\theta 1}=1$. Natural leaves are "most of the time" vertical. Following the proposal used in canopy radiative transfer studies, the inclination distribution is assumed to be erectophile [11], so $K_{\theta 1}=0.85$.

\subsubsection{Sedimentation}

The single leaf deposition velocity, $v^{S}$, depends on the settling velocity and the leaf inclination

$$
v^{S}=\frac{1}{2} W_{S} \cos \theta
$$

The inclination angle is the only random parameter, so the macroscopic deposition velocity is deduced from Eq. (6):

$$
V^{S}=\frac{1}{2} W_{S} K_{\theta 2}
$$

where $K_{\theta 2}$ is a constant linked to the inclination distribution by $K_{\theta 2}=\overline{\cos \theta}$. For artificial grass, $K_{\theta 2}=0$ and for erectophile natural grass, $K_{\theta 2}=0.42$.

\subsubsection{Brownian diffusion}

Brownian diffusion transfer and deposition depend on leaf boundary layer characteristics. Simple Reynolds number calculations let us suppose that the latter is laminar. Single leaf deposition velocity is then expressed [12] by

$$
v^{B}=0.664 D_{B}^{2 / 3} \frac{U^{1 / 2}}{L^{1 / 2} v_{a}^{1 / 6}}
$$

where $D_{B}$ is the Brownian diffusivity and $v_{a}$ the kinematic viscosity. The leaf width is the only random parameter. Its distribution is supposed to be uniform. Applying (6), the macroscopic deposition velocity is:

$$
V^{B}=0.563 D_{B}^{2 / 3} \frac{U_{e}^{1 / 2}}{\bar{L}^{1 / 2} v_{a}^{1 / 6}}
$$

The model has been applied to three deposition processes. With the deduced practical formulations of macroscopic deposition velocities, it is possible to compare model and experimental results. 


\subsection{Results}

\subsubsection{Lycopodium spores deposition}

Chamberlain [9] has investigated the deposition of Lycopodium spores on grass. The concentration profile has not been measured over the entire domain, but only at two heights, namely $z_{R}$ and $z_{1}=4 / 3 h_{c}$. Their ratio is given for different friction velocity values and can be compared with the model results. Measurements and model results for artificial grass are shown on figure 1.

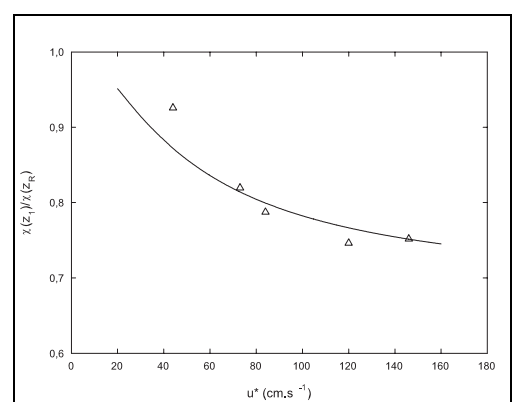

Figure 1. Measured (triangles) and modelled (solid line) concentration ratios on artificial grass as a function of the friction velocity.

The proposed model reproduces well the decreasing tendency of the measured concentration ratios with increasing friction velocity. At low friction velocity, discrepancy grows, but this is probably due to inaccurate concentration measurement methods. Indeed the latter is based on impacting particles counting. Statistical problems may occur at low friction velocity, when this process is not effective.

The other measurements set concerns the canopy deposition velocity (9). Model and experimental results are shown on figure 2 for both artificial and natural grasses.

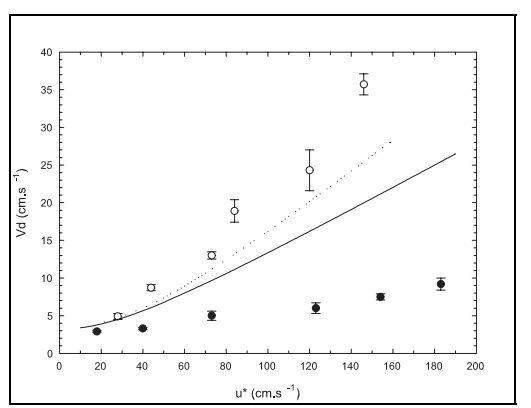

Figure 2. Measured and modelled deposition velocities over artificial and natural grasses. Measurement symbols are respectively empty and full circles, while the model results are represented through dotted (artificial) and solid (natural) lines.

For artificial sticky grass, model underestimates measurements, discrepancy increasing with increasing friction velocity. It lies under $40 \%$ over the studied friction velocity range. It is possible that turbulent impaction has some influence on Lycopodium deposition at high friction velocity. However agreement is satisfactory. For natural grass, model overestimates measurements, up to a factor 3 at high friction velocity. In order to explain this discrepancy for natural surfaces, model hypotheses are to be criticized. Vegetated surfaces are considered as perfect sink. But it is known that large aerosols (typically larger than 5 or $10 \mu \mathrm{m}$ ) under strong wind conditions are susceptible to rebound from non sticky surfaces [13]. 


\subsubsection{Aerosol size dependence of deposition velocity}

To conclude this comparison with Chamberlain's experiments, the model is applied to smaller aerosols for exploring the aerosol size influence on deposition velocity.

Three different flow regimes are investigated, $\mathrm{u}^{*}=35,70$ and $140 \mathrm{~cm} . \mathrm{s}^{-1}$. The measurements and model results are shown on figure 3 .

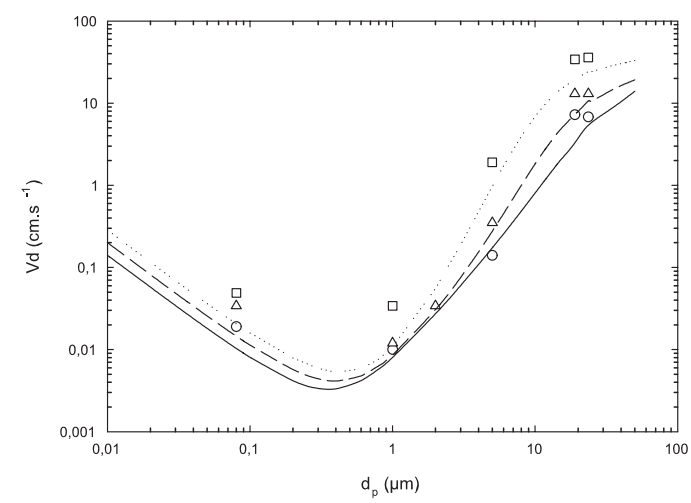

a) Artificial grass

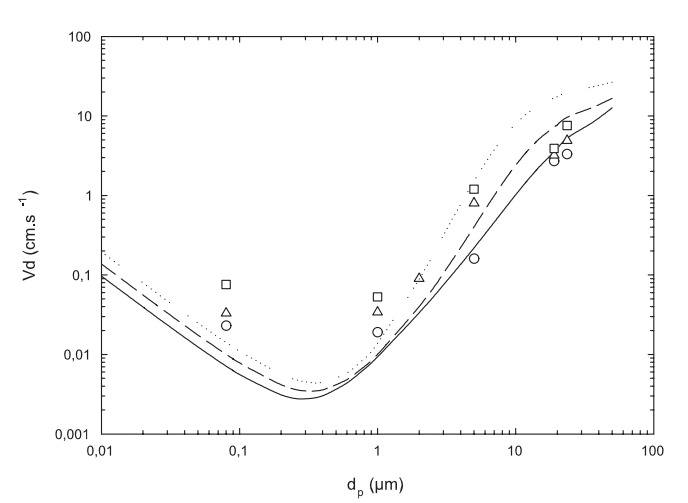

b) Natural grass

Figure 3. Deposition velocity over grass at $u^{*=} 35,70$ and $140 \mathrm{~cm} \cdot \mathrm{s}^{-1}$. The corresponding measurement symbols are respectively circles, squares and triangles. Model results are plotted as solid, long dash and dotted lines.

The model reproduces fairly well the deposition velocity evolution over four decades of size variation, especially for artificial grass. Micronic and sub-micronic aerosols seem to deposit better on natural grass than on artificial, while model results are quite similar. Following Chamberlain's proposed explanation, they could be more sensitive to surface structure than to surface stickiness, as were 20 micron aerosols. In this context, interception could be an important process for sub-micronic aerosols.

\section{CONCLUSIONS}

Our modelling approach has been applied to three important deposition mechanisms. Transport equation has been simplified. The resulting model has been implemented and applied to a grass experiments campaign in a wind tunnel [9]. The model reproduces well the behaviour of 20 micron aerosols depositing on artificial grass. A better fit could be achieved if turbulent impaction was addressed. On natural grass an important discrepancy is observed. Bounce-off is suspected to have a strong influence on deposition of large aerosols. The aerosol size influence is reasonably well caught. In order to achieve a better fit, secondary aerosol phenomena should be included, particularly interception, which seems to be an efficient phenomenon for sub-micronic aerosols. The model validation next step will be realized on forest canopy configuration.

\section{Acknowledgments}

The author is grateful to A. Mailliat (IRSN, Cadarache), F. Anselmet and M. Amielh (IRPHE, Marseille) and M.-A. Gonze (IRSN, Cadarache) for scientific support. 


\section{References}

[1] Ogorodnikov B. I., Budyka A. K. and Skitovitch V.I., J. Aerosol Sci. 25 (1994) 269-270.

[2] Slinn W. G. N., Atmos. Environ. 16 (1982) 1785-1794.

[3] Gallagher M. W., Beswick K.M., Duyzer J.H., Westrate H., Choularton T.W. and Hummelshoj P., Atmos. Environ. 31 (1997) 359-373.

[4] Wyers G. P. and Duyzer J. H., Atmos. Environ. 31 (1997) 333-343.

[5] Ould-Dada Z., Sci. Total Environ. 286 (2002) 83-96.

[6] Finnigan J. J., Annu. Rev. Fluid Mech. 32 (2000) 519-571.

[7] Raupach M. R., Coppin P.A. and Legg B.J. Bound.-Layer Meteorol. 35 (1986) 21-52.

[8] Petroff A., "Etude phénoménologique du dépôt sec d'aérosols sur un couvert végétal", XIX ${ }^{\text {th }}$ French Aerosol Congress, Paris 2003, ASFERA.

[9] Chamberlain A. C., Proc. R. Soc. Lond., Ser. A 296 (1967) 45-70.

[10] Belot Y., Baille A. and Delmas J.-L., Atmos. Environ. 10 (1976) 89-98.

[11] Goel N. S. and Strebel D. E. Agron. J. 76 (1984) 800-802.

[12] Polhausen E., Z. Angew. Math. Mech. 1 (1921) 115-121.

[13] Chamberlain, A. C. in Vegetation and the Atmosphere, Monteith, J. L. ed (Academic Press, London, 1975) pp. 155-228. 